$\mathbf{R}$

\title{
Relationship between Autoimmune Pancreatitis and Pancreatic Cancer: A Single-Center EXPERIENCE
}

Tsukasa Ikeura, MD, PhD, Hideaki Miyoshi, MD, PhD, Kazushige Uchida, MD, PhD, Toshiro Fukui, MD, PhD, Masaaki Shimatani, MD, PhD, Yuri Fukui, MD, PhD, Kimi Sumimoto, MD, Mitsunobu Matsushita, MD, PhD, Makoto Takaoka, MD, PhD, Kazuichi Okazaki, MD, PhD.

The third Department of Internal Medicine, Kansai Medical University, Osaka, Japan

Running title: Relationship between AIP and pancreatic cancer

Keywords: autoimmune pancreatitis, pancreatic cancer, chronic pancreatitis, carcinogenesis

Disclosures: The authors declare that there is no conflict of interest.

Address for correspondence: Tsukasa Ikeura

The Third Department of Internal Medicine

Kansai Medical University

2-3-1, Shinmachi, Hirakata

573-1191 Osaka - Japan

Phone: +81-72-804-0101

Fax:+81-72-804-2524

E-mail: ikeurat@takii.kmu.ac.jp

\section{AUTHOR CONTRIBUTIONS:}

The contributions of the Authors are the following:

1. Conception and design of the study: Tsukasa Ikeura, Hideaki, Miyoshi, Yuri Fukui, and Kazuichi Okazaki

2. Collection of data: Tsukasa Ikeura, Hideaki Miyoshi, Kazushige Uchida, Toshiro Fukui, Masaaki Shimatani, Yuri Fukui, Kimi Sumimoto, and Makoto Takaoka

3. Drafting: Tsukasa Ikeura, Hideaki Miyoshi, and Yuri Fukui

4. Revision of the manuscript; Kazushige Uchida, Mitsunobu Matsushita, and Kazuichi Okazaki

5. Approval of the final version of the manuscript: Kazuichi Okazaki 


\section{ABSTRACT}

Objectives: Ordinary chronic pancreatitis $(\mathrm{CP})$, such as alcoholic $\mathrm{CP}$, is well established to have the increased risk for pancreatic cancer $(\mathrm{PaC})$, nevertheless an association between autoimmune pancreatitis (AIP) and $\mathrm{PaC}$ is still unknown. The aims of this study are to examine the frequency of patients who developed $\mathrm{PaC}$ during follow-up after being diagnosed with type $1 \mathrm{AIP}$ and to compare the incidence rate of $\mathrm{PaC}$ between patients with type $1 \mathrm{AIP}$ and $\mathrm{CP}$.

Methods: Sixty-three patients with type 1 AIP and 41 patients with $\mathrm{CP}$ were enrolled. We examined development of PaC during follow-up from their clinical records.

Results: The mean follow-up period was 62.4 months in AIP group and 49.2 months in CP group. The occurrence of $\mathrm{PaC}$ was observed in 3 patients with AIP during the mean follow-up period of 94.7 months (range, 31-186), whereas a single CP patient developed PaC 38 months after CP diagnosis. The incident rate of $\mathrm{PaC}$ during follow-up was comparable between the 2 groups [4.8\% (3/63) in type 1 AIP group vs. 2.4\% (1/41) in CP group]. In all of 3 AIP patients who developed accompanying PaC, the clinical remission of AIP was achieved with maintenance steroid therapy, when tumors were discovered. In the histological examination of one surgical patient with $\mathrm{PaC}$, lymphoplasmacytic infiltration in storiform fibrosis with 
abundant IgG4-positive cell infiltration was observed around the $\mathrm{PaC}$ area.

Conclusions: Similar to patients with ordinary $\mathrm{CP}$, surveillance for development of $\mathrm{PaC}$ is needed at regular interval during follow-up in AIP patients. 


\section{INTRODUCTION}

Autoimmune pancreatitis (AIP), which was first proposed as a clinical entity by Yoshida et al. in

1995, is characterized by focal or diffuse pancreatic enlargement, irregular narrowing of the main pancreatic duct (MPD), and a dramatic response to steroid therapy ${ }^{1-4}$. To date, 2 AIP subtypes have been recognized, type 1 and type $2^{5}$ The histological features of type 1 AIP include periductal infiltration of lymphocytes, abundant IgG4-positive plasma cells, storiform fibrosis, and obliterative phlebitis, specifically lymphoplasmacytic sclerosing pancreatitis (LPSP). Type 2 AIP, on the other hand, is histologically characterized by the presence of granulocytic epithelial lesions without IgG4-positive plasma cell infiltration, specifically idiopathic duct-centric chronic pancreatitis (IDCP) or AIP with granulocyte epithelial lesions $(\mathrm{GELs})^{6,7}$. Previous studies have reported the distinguishing characteristics in clinical profiles and outcomes of type 1 and type $2 \mathrm{AIP}^{8-12}$. Type 1 AIP is classified as a pancreatic manifestation of IgG4-related disease, and is probably a systemic disease with an abnormal immunological process ${ }^{13}$.

Currently, little is known about the natural clinical history of AIP. The prognosis of AIP is considered to be favorable because it is responsive to steroid therapy. However, reports indicate that $24-57 \%$ of the patients who receive steroid therapy experience a relapse $\mathrm{e}^{13-15}$. Additionally, previous papers 
have suggested an association between AIP and pancreatic cancer $(\mathrm{PaC})^{16-24}$. It is extremely important for clinicians to recognize the validity and the magnitude of the association between AIP and PaC, although whether patients suffering from AIP are more susceptible to $\mathrm{PaC}$ has not been clearly elucidated.

The aims of this study were to examine the frequency with which patients developed PaC during follow-up after being diagnosed with type 1 AIP, to clarify the clinical and histological characteristics of type 1 AIP patients with $\mathrm{PaC}$, and to compare the incidence rate of $\mathrm{PaC}$ between patients with type 1 AIP and ordinary chronic pancreatitis $(\mathrm{CP})$, which is a well-established risk factor for $\mathrm{PaC}^{25,26}$.

\section{METHODS}

\section{Identification of type 1 AIP patients}

We reviewed data from all AIP patients included in our database starting in 2002. We applied the

international consensus diagnostic criteria (ICDC) to each patient based on clinical, serological, radiological, and histological findings ${ }^{5}$. The current study included patients diagnosed with type 1 AIP who were followed for more than 1 year using imaging modalities. These criteria led to inclusion of 63 patients suffering from type 1 AIP (definitive type 1 AIP in 61 patients, and probable type 1 AIP in 2 patients) in the 
study. Many of these patients were included in the previously published papers ${ }^{22,27,28}$.

For the purpose of this study, we used clinical records to retrieve patient data, including age at the clinical onset of the disease, sex, length of observation period, and development of PaC or extrapancreatic cancer during the follow-up period. The observation period was defined as the time from the diagnosis of AIP until the final contact or, in cases accompanied by $\mathrm{PaC}$, until the diagnosis of $\mathrm{PaC}$. To allow further analysis of the clinical factors associated with development of $\mathrm{PaC}$ in type $1 \mathrm{AIP}$ patients, we also collected the following information: alcohol and smoking habits, medical history, diabetes history, pancreatic parenchymal finding, pancreatic ductal finding, IgG4 serum levels, other organ involvement, therapy for the disease (steroid, resection, no treatment), and disease relapse.

\section{Identification of ordinary CP patients}

Clinical information of ordinary CP patients, including alcoholic CP and hereditary CP patients, who

were observed at our institution from November 2002 to December 2011, were retrospectively reviewed to compare the incidence rate of $\mathrm{PaC}$ between patients with type $1 \mathrm{AIP}$ and patients with ordinary CP. Of these $\mathrm{CP}$ patients, only $\mathrm{CP}$ patients who were followed for more than 1 year using imaging modalities were included in the current study. The diagnosis of CP was based on the clinical diagnostic criteria proposed by 
the Japan Pancreas Society ${ }^{29}$. We enrolled 41 CP patients (34 with definitive CP and 7 with probable CP).

Briefly, 34 patients diagnosed as definitive $\mathrm{CP}$ had multiple calcifications distributed in the entire pancreas

on computed tomography (CT), and 7 patients diagnosed as probable $\mathrm{CP}$ had no calcification in the pancreas but irregular dilatation of the MPD throughout the entire pancreas plus pancreatic deformity with irregular contour on CT. As with type 1 AIP patients, clinical information of included CP patients was reviewed.

\section{Definitions}

Patients were divided into 2 groups based on their alcohol consumption: (1) teetotalers (non-drinkers),

and (2) drinkers. They were also divided on the basis of smoking habits into non-smokers and smokers.

Diabetes was defined as a fasting glucose level > $126 \mathrm{mg} / \mathrm{dL}$ or a glucose level > $201 \mathrm{mg} / \mathrm{dL} 2$ hours

after an oral glucose tolerance test.

The upper limit of normal value of serum IgG4 was defined as $135 \mathrm{mg} / \mathrm{dL}^{30}$.

Initial steroid therapy was performed with either conventional oral steroid therapy or steroid pulse therapy $^{31,32}$. For conventional oral steroid therapy, prednisolone was administrated at an initial dose of 30 to $40 \mathrm{mg}$ per day for 2 weeks, and was subsequently gradually tapered by $5 \mathrm{mg}$ every 1 to 2 weeks until the 
maintenance dosage was reached. Steroid pulse therapy involved intravenous administration of methylprednisolone at a dose of $500 \mathrm{mg}$ per day for 3 consecutive days per week for 2 straight weeks. After steroid pulse therapy, we administered prednisolone at $20 \mathrm{mg}$ per day, and it was gradually tapered until the maintenance dosage was reached. After initial steroid therapy, if needed, maintenance steroid therapy was performed for at least 6 months with prednisolone at a dose of 2.5 to $5.0 \mathrm{mg}$ per day to prevent relapse.

AIP relapse was defined as the reappearance of pancreatic or extrapancreatic involvement after steroid withdrawal.

\section{Statistical analysis}

Differences among the groups were analyzed using chi-squared test or Fisher's Exact test for qualitative variables, and Mann-Whitney's U test for quantitative variables. A p-value > 0.05 was considered statistically significant.

\section{RESULTS}

\section{Characteristics of type 1 AIP Patients}


The characteristics of the 63 patients with type 1 AIP are shown in Table 1. The mean follow-up

period was 62.4 months (range, 12-195 months). All patients underwent imaging modalities, such as CT and magnetic resonance cholangiopancreatography (MRCP), for surveillance of AIP at least once a year during follow-up.

CT at the time of diagnosis of AIP revealed diffuse enlargement of the pancreas in 26 patients (41\%), focal enlargement in 30 (48\%), and a pancreatic mass without parenchymal enlargement in 7 (11\%). Examination with endoscopic retrograde cholangiopancreatography (ERCP) or MRCP (available in 57 patients) revealed long or multiple strictures of the MPD in 30 patients (53\%), focal narrowing of the MPD in 22 patients (39\%), and no narrowing of the MPD in 5 patients $(8 \%)$. IgG4 serum levels were measured in 61 patients at the time of diagnosis of AIP. IgG4 serum levels were higher than twice the upper normal limit in 40 patients $(66 \%), 1$ to 2 times the upper normal limit in 17 patients $(28 \%)$, and normal in 4 patients $(6 \%)$. Twenty patients $(31 \%)$ had other organ involvement [biliary duct stricture in 9 patients (14\%), enlargement of the salivary or lachrymal gland in $7(11 \%)$, retroperitoneal fibrosis in $5(8 \%)$, and renal involvement in 3 $(5 \%)]$. Fifty-one of the 63 patients $(81 \%)$ were treated with steroid therapy (31 with conventional oral steroid therapy and 20 with steroid pulse therapy) as the initial treatment for AIP. After initial steroid therapy, 39 patients underwent maintenance steroid therapy. All patients who received steroid therapy 
showed remission of the disease. Seven out of 63 patients $(11 \%)$ underwent surgery because of suspected

cancer. The remaining 5 patients $(8 \%)$ were followed-up conservatively. Of 59 patients who underwent

surgery or steroid therapy, 12 patients (19\%) exhibited relapse of the disease.

Of 63 patients, 8 cancers were observed in 6 patients (9\%), either synchronously or metachronously.

The spectrum of malignancies included $\mathrm{PaC}(\mathrm{n}=3)$, gastric cancer $(\mathrm{n}=2)$, colon cancer $(\mathrm{n}=1)$, leukemia $(\mathrm{n}=1)$, and melanoma $(\mathrm{n}=1)$. All PaCs were found after the diagnosis of AIP.

\section{Development of $\mathrm{PaC}$ in type 1 AIP patients}

Case 1

A 61-year-old female presented with a mass-like enlargement of the pancreatic head with focal narrowing of the MPD (Fig. 1a). Her IgG4 serum level was $338 \mathrm{mg} / \mathrm{dL}$. She was diagnosed with AIP based on these findings. Initial steroid therapy and subsequent maintenance therapy with prednisolone were performed and led to remission of the disease (Fig. 1b). Thirty-one months after the AIP diagnosis, she experienced jaundice due to a stricture in the lower bile duct despite being on maintenance steroid therapy.

CT showed a low-density irregular mass in the pancreatic head (Fig. 1c), which endoscopic ultrasound-guided fine-needle aspiration (EUS-FNA) showed was adenocarcinoma. She underwent 
pancreatoduodenectomy, and histological examination of the resected pancreata revealed a moderately

differentiated ductal adenocarcinoma in the pancreatic head (Fig. 2a). Marked lymphoplasmacytic

infiltration with storiform fibrosis surrounded the cancerous region (Fig. 2b). Immunohistochemical

analysis revealed numerous IgG4-expressing plasma cells in the inflammatory area (Fig. 2c). Pancreatic intraepithelial neoplasia (PanIN) lesion was not seen in the non-cancerous region surrounding the PaC (Fig. 2d). After surgery, no morphological change was seen in the remnant pancreas on CT.

Case 2

A 39-year-old woman was diagnosed with AIP based on elevated serum levels of IgG4 and radiological abnormalities in the pancreatic tail such as focal enlargement of the pancreas and narrowing of the MPD. She had other autoimmune disorders, such as mixed connective tissue disease and Sjogren's syndrome. She underwent maintenance steroid therapy, which led to remission of the disease. One hundred eighty-six months after the diagnosis of AIP, a low-density mass was identified in the pancreatic body on contrast-enhanced CT performed for surveillance of AIP. EUS-FNA was used to obtain a sample from the mass, and the result of the cytological examination was positive for malignancy. She underwent distal pancreatectomy. Histological examination revealed a moderately differentiated ductal adenocarcinoma in the pancreatic body (Fig. 3a). The acinar cells around the cancerous region were replaced by massive inter- 
and intralobular fibrosis with a pattern that was not storiform fibrosis (Fig. 3b). Obliterative phlebitis was

not seen. Immunohistochemistry revealed scattered IgG4-positive cells (Fig. 3c). PanIN lesion was not seen in the non-cancerous region surrounding the $\mathrm{PaC}$ (Fig 3d).

Case 3

An 80-year-old male had obstructive jaundice due to a focal enlargement in the head of the pancreas

(Fig. 4a). ERCP showed diffuse narrowing of the MPD. His IgG4 serum levels were $154 \mathrm{mg} / \mathrm{dL}$. Based on the diagnosis of AIP, he was treated with a steroid, and it led to improvement of clinical and radiological findings (Fig. 4b). While being treated with maintenance steroid therapy (67 months after the diagnosis of AIP), a pancreatic body tumor with upstream dilatation of the pancreatic duct was detected (Fig. 4c). Cytological examination of material obtained by EUS-FNA from the tumor revealed adenocarcinoma, and the laparoscopic study identified peritoneal metastasis, leading to a diagnosis of unresectable PaC. He was treated with systemic chemotherapy.

\section{Comparison of clinical characteristics between patients with or without $\mathrm{PaC}$}

There were no significant differences in age, observation period, incidence of drinking and smoking, or prevalence of diabetes between patients who developed and those who did not develop PaC (Table 2). A 
higher proportion of patients who developed $\mathrm{PaC}$ had focal enlargement of the pancreas, focal narrowing of the MPD, and marked elevation in IgG4 levels compared with patients who did not develop PaC, but there were no significant differences between the 2 groups. The percentage of type 1 patients who underwent initial steroid therapy and subsequent maintenance steroid therapy was comparable between the 2 groups.

\section{Comparison in incidence of $\mathrm{PaC}$ between type 1 AIP patients and $\mathrm{CP}$ patients}

Forty-one patients were included in CP group [29 males and 12 females; mean age at clinical onset, 60.2 years (range, 22-89)] (Table 3). The percentage of males, age at clinical onset, and observation period were comparable between CP group and type 1 AIP group. There was a statistically significant difference in the prevalence of alcohol drinkers between the 2 groups ( $8 \%$ in type 1 AIP group vs. $61 \%$ in CP group, p < 0.001). PaC occurred in a single patient 38 months after $\mathrm{CP}$ diagnosis. The incident rate of $\mathrm{PaC}$ in type 1 AIP group was $4.8 \%(3 / 63)$, which was higher than in CP group [2.4\% (1/41)], although there was no statistical difference. The annual incidence rates of $\mathrm{PaC}$ in type $1 \mathrm{AIP}$ group and $\mathrm{CP}$ group were $0.92 \%$ and $0.59 \%$ per year, respectively. 


\section{DISCCUSION}

This is the first paper to systematically assess the prevalence of $\mathrm{PaC}$ in patients with type $1 \mathrm{AIP}$. The

results show that $\mathrm{PaC}$ occurred in $4.8 \%$ of type 1 AIP patients during the observation period. This incident

rate was comparable to that of patients suffering from ordinary $\mathrm{CP}(2.4 \%)$.

Chronic inflammatory processes play a well-recognized role in carcinogenesis: e.g., liver cancer in chronic hepatitis $\mathrm{B}$ and $\mathrm{C}^{33}$, gastric cancer in Helicobacter pylori-induced gastritis ${ }^{34}$, and colon cancer in inflammatory bowel disease ${ }^{35}$. Additionally, ordinary $\mathrm{CP}$, such as alcoholic, hereditary, and tropical CP, has been established to increase the risk for developing $\mathrm{PaC}$ due to progressive and irreversible inflammation of the pancreas. Pre-existing ordinary CP result in about a 10-20-fold increased risk of pancreatic cancer ${ }^{25,26}$. The previously held belief was that AIP, in contrast to $\mathrm{CP}$, is a reversible disease with a favorable prognosis because of the dramatic improvement of clinical and radiological findings when steroid therapy is given.

However, persistently high IgG4 serum concentrations and relapses of pancreatic swelling and narrowing of the MPD occur in $60 \%$ and $58 \%$ of AIP patients, respectively, after steroid therapy, indicating that chronic inflammation of the pancreas in AIP patients may continue histologically even after remission of the disease is induced by steroid therapy ${ }^{36,37}$. Indeed, a recent paper reported that type 1 AIP patients with 
pancreatic head swelling or MPD nonnarrowing in the pancreatic body may progress to an advanced stage

of $\mathrm{CP}$ due to pancreatic juice stagnation ${ }^{38}$. As biologic evidence that supports an association between AIP

and $\mathrm{PaC}$, the prevalence of PanIN in AIP patients was reported to be higher than that in non-autoimmune

forms of pancreatitis, although this comparison did not reach the level of statistical significance. Moreover,

significant K-ras mutations, which are known to be found in nearly all patients with pancreatic cancer, were

frequently detected in the pancreas of patients suffering from $\mathrm{AIP}^{17,39}$. Taking the above into consideration,

AIP might have malignant potential for development of $\mathrm{PaC}$, as has been observed with ordinary $\mathrm{CP}$.

A recent study by Shiokawa et al. reported that the risk of developing various cancers was highest

during the first year after AIP diagnosis, and that most patients diagnosed with cancer prior to the diagnosis

of AIP did not experience relapse of AIP after the successful treatment of the cancer ${ }^{40}$. From these data, they

speculate that AIP may be a manifestation of paraneoplastic syndrome, which is a rare condition triggered

by an altered immune system response to a neoplasm ${ }^{41}$. In our cohort, 3 PaCs were found during the mean

follow-up period of 94.7 months (range, 31-186 months), indicating that the process of carcinogenesis had

not yet occurred in the pancreas at the time of the diagnosis of type 1 AIP, and that our cases were not

associated with paraneoplastic syndrome. 
Although case reports and studies regarding synchronous or metachronous development of $\mathrm{PaC}$ in

AIP patients have been published before, there have been only a few papers that have used resected pancreatic tissue to examine the histological association between $\mathrm{PaC}$ and the parenchyma surrounding the $\mathrm{PaC}^{23}$, 24. This is because patients with $\mathrm{PaC}$ frequently have unresectable disease due to late clinical presentation. In this study, we were able to use resected pancreata to histologically evaluate in detail 2 AIP patients who developed accompanying $\mathrm{PaC}$. One patient (Case 1) demonstrated not only dense lymphoplasmacytic infiltration in storiform fibrosis, but also infiltration of numerous IgG4-positive plasma cells around the PaC, fulfilling histological Level 1 Criteria for type 1 AIP according to ICDC ${ }^{5}$ This finding that PaC occurred in the background of LPSP suggested that carcinogenesis can be attributed to longstanding pancreatic inflammation. The other patient (Case 2) exhibited dense fibroinflammation with acinar atrophy accompanied by rather mild inflammatory infiltration around the PaC area. IgG4 immunostaining of this area showed scanty IgG4-positive cell infiltration. These changes were consistent with those observed in obstructive pancreatitis caused by $\mathrm{PaC}^{42}$. Therefore, the process of pancreatic carcinogenesis in the 2 cases appears to be different. On the other hand, Gupta et al. reported that the prevalence of PanIN in type 1 AIP and non-autoimmune forms of CP was $64 \%$ and $63 \%$, respectively, suggesting the possibility that the risk of malignancy in type 1 AIP and ordinary CP may be equivalent. 
However, PanIN lesion was not seen in the non-cancerous region surrounding the $\mathrm{PaC}$ in these 2 patients.

Further histological investigation about pancreatic carcinogenesis in AIP is needed.

Three multicenter surveys reported that $\mathrm{PaC}$ occurred less commonly after the diagnosis of AIP,

which is in contrast to the result of our single-center study ${ }^{10,13,43}$. The most recent study also demonstrated

that patients with AIP had a high risk of developing various cancers, such as gastric cancer, prostate cancer,

and colon cancer, but none of the AIP patients had $\mathrm{PaC}^{40}$. Although the incidence rate for the development

of $\mathrm{PaC}$ in AIP patients might be overestimated in this study owing to population bias, we should be careful

to monitor for the development of $\mathrm{PaC}$ during long-term follow-up.

Interestingly, all 3 AIP patients who developed $\mathrm{PaC}$ were recognized as focal type AIP at the onset

of the disease and receiving low-dose maintenance therapy with prednisolone at the diagnosis of PaC. The

previous study about clinical differences between the focal and diffuse AIP reported that the mean serum

levels of IgG4 were significantly higher in focal than in diffuse AIP, and that recurrences occurred more

frequently in focal AIP than in diffuse $\mathrm{AIP}^{3}$. This data may suggest the possibility that the pancreatic

inflammation may be more likely to be sustained even after steroid therapy in focal type AIP more than in

diffuse type AIP. From the standpoint that chronic inflammatory processes play a critical role in carcinogenesis, patients with focal type AIP may be likely to develop PaC. Moreover, the 
immunosuppressed state of the patients that is produced by longstanding steroid therapy is also possibly

related to the development of $\mathrm{PaC}$ in AIP patients ${ }^{44}$. However, in the comparison between AIP patients with

and those without $\mathrm{PaC}$, focal form of the disease and maintenance steroid therapy were not statistically

identified as a clinical predictor for the development of $\mathrm{PaC}$ in AIP patients. This may be because the study

sample size was small. Larger prospective studies are needed to clarify whether or not focal form of the

disease and maintenance steroid therapy are closely related to carcinogenesis of the pancreas in AIP.

Diagnosing AIP can be challenging, because AIP and $\mathrm{PaC}$ share clinical and radiological

characteristics, such as age of disease onset, mass formation in the pancreas, and painless obstructive

jaundice. While AIP is responsive to steroid therapy, surgical resection is the only curative treatment for

$\mathrm{PaC}^{45}$. Therefore, it is extremely crucial to differentiate between $\mathrm{AIP}$ and $\mathrm{PaC}$ in order to avoid

inappropriate treatment. In the patients who had AIP accompanied by $\mathrm{PaC}$, tumors were incidentally

discovered as new findings, such as mass formation and stricture of the lower bile duct, on CT or MRCP

performed as surveillance for AIP. These findings in imaging studies of AIP patients during follow-up may

lead to a mistaken diagnosis of relapsed AIP. In order to avoid inappropriate treatment, it is important to

recognize the possibility that $\mathrm{PaC}$ can develop in AIP patients during the observation period, and

pathological examination using EUS-FNA is needed. However, there may also be a concern that patients 
with PaC may be misdiagnosed with AIP due to insufficient biopsy specimens by fine needle aspiration,

and because pancreatic cancer sometimes shows histological findings mimicking those of AIP, such as

marked infiltration with IgG4-positive plasma cells ${ }^{46-48}$. Furthermore, in steroid trials that use conventional

oral steroid therapy or steroid pulse therapy, it is essential to confirm the partial or complete resolution of the disease with tests for radiological and serological abnormalities, even after a negative workup for cancer by EUS-FNA ${ }^{5,32,49}$.

In conclusion, patients with type $1 \mathrm{AIP}$ have a higher risk of $\mathrm{PaC}$, similar to patients with ordinary $\mathrm{CP}$, although the result we offered was not sufficient to categorically link type 1 AIP with an elevated risk of carcinoma. Nonetheless, surveillance not only for relapse of AIP but also for development of PaC is needed at regular intervals during follow-up of type 1 AIP patients. Long-term prospective studies in large cohorts of AIP patients are required to determine the exact relationship between AIP and $\mathrm{PaC}$.

\section{ACKNOWLEDGMENT}

The authors thank Dr. Sakagami Junichi, Kyoto Prefectural University of Medicine, for providing clinical data of a patient included in this study.

This study was partly supported by (1) Grant-in-Aid for Scientific Research of Ministry of Culture and 
Science of Japan (23591017,24591020), (2) Grant-in-Aid for "Research for Intractable Disease" Program

from the Ministry of Health, Labour and Welfare of Japan, (3) grants-in-aid from CREST Japan Science and Technology Agency, and (4) NEXT-Supported Program for the Strategic Research Foundation at Private Universities. 
1. Yoshida K, Toki F, Takeuchi T, Watanabe S, Shiratori K, Hayashi N. Chronic pancreatitis caused by an autoimmune abnormality. Proposal of the concept of autoimmune pancreatitis.

Dig Dis Sci 1995;40:1561-8.

2. Finkelberg DL, Sahani D, Deshpande V, Brugge WR. Autoimmune pancreatitis. N Engl J Med 2006;355:2670-6.

3. Frulloni L, Scattolini C, Falconi M, Zamboni G, Capelli P, Manfredi R, et al. Autoimmune pancreatitis: differences between the focal and diffuse forms in 87 patients. Am J Gastroenterol 2009;104:2288-94.

4. Okazaki K, Uchida K, Koyabu M, Miyoshi H, Takaoka M. Recent advances in the concept and diagnosis of autoimmune pancreatitis and IgG4-related disease. $\mathbf{J}$ Gastroenterol 2011;46:277-288.

5. Shimosegawa T, Chari ST, Frulloni L, Kamisawa T, Kawa S, Mino-Kenudson M, et al. International consensus diagnostic criteria for autoimmune pancreatitis: guidelines of the International Association of Pancreatology. Pancreas 2011;40:352-8.

6. Notohara K, Burgart LJ, Yadav D, Chari S, Smyrk TC. Idiopathic chronic pancreatitis with 
periductal lymphoplasmacytic infiltration: clinicopathologic features of 35 cases. Am J Surg

Pathol 2003;27:1119-27.

7. Zamboni G, Luttges J, Capelli P, Frulloni L, Cavallini G, Pederzoli P, et al. Histopathological features of diagnostic and clinical relevance in autoimmune pancreatitis: a study on 53 resection specimens and 9 biopsy specimens. Virchows Arch 2004;445:552-63.

8. Sah RP, Chari ST, Pannala R, Sugumar A, Clain JE, Levy MJ, et al. Differences in clinical profile and relapse rate of type 1 versus type 2 autoimmune pancreatitis. Gastroenterology 2010;139:140-8; quiz e12-3.

9. Maire F, Le Baleur Y, Rebours V, Vullierme MP, Couvelard A, Voitot H, et al. Outcome of patients with type 1 or 2 autoimmune pancreatitis. Am J Gastroenterol 2011;106:151-6.

10. Kamisawa T, Kim MH, Liao WC, Liu Q, Balakrishnan V, Okazaki K, et al. Clinical characteristics of 327 Asian patients with autoimmune pancreatitis based on Asian diagnostic criteria. Pancreas 2011;40:200-5.

11. Detlefsen S, Zamboni G, Frulloni L, Feyerabend B, Braun F, Gerke O, et al. Clinical features and relapse rates after surgery in type 1 autoimmune pancreatitis differ from type 2: a study of 114 surgically treated European patients. Pancreatology 2012;12:276-83. 
12. Okazaki K, Uchida K, Ikeura T, Takaoka M. Current concept and diagnosis of IgG4-related disease in the hepato-bilio-pancreatic system. J Gastroenterol 2013;48:303-14.

13. Kamisawa T, Shimosegawa T, Okazaki K, Nishino T, Watanabe H, Kanno A, et al. Standard steroid treatment for autoimmune pancreatitis. Gut 2009;58:1504-7.

14. Kubota K, Watanabe S, Uchiyama T, Kato S, Sekino Y, Suzuki K, et al. Factors predictive of relapse and spontaneous remission of autoimmune pancreatitis patients treated/not treated with corticosteroids. J Gastroenterol 2011;46:834-42.

15. Sandanayake NS, Church NI, Chapman MH, Johnson GJ, Dhar DK, Amin Z, et al. Presentation and management of post-treatment relapse in autoimmune pancreatitis/immunoglobulin G4-associated cholangitis. Clin Gastroenterol Hepatol 2009;7:1089-96.

16. Gupta R, Khosroshahi A, Shinagare S, Fernandez C, Ferrone C, Lauwers GY, et al. Does autoimmune pancreatitis increase the risk of pancreatic carcinoma?: a retrospective analysis of pancreatic resections. Pancreas 2013;42:506-10.

17. Kamisawa T, Tsuruta K, Okamoto A, Horiguchi S, Hayashi Y, Yun X, et al. Frequent and significant K-ras mutation in the pancreas, the bile duct, and the gallbladder in autoimmune pancreatitis. Pancreas 2009;38:890-5. 
18. Loos M, Esposito I, Hedderich DM, Ludwig L, Fingerle A, Friess H, et al. Autoimmune pancreatitis complicated by carcinoma of the pancreatobiliary system: a case report and review of the literature. Pancreas 2011;40:151-4.

19. Ghazale A, Chari S. Is autoimmune pancreatitis a risk factor for pancreatic cancer? Pancreas $2007 ; 35: 376$

20. Inoue H, Miyatani H, Sawada Y, Yoshida Y. A case of pancreas cancer with autoimmune pancreatitis. Pancreas 2006;33:208-9.

21. Pezzilli R, Vecchiarelli S, Di Marco MC, Serra C, Santini D, Calculli L, et al. Pancreatic ductal adenocarcinoma associated with autoimmune pancreatitis. Case Rep Gastroenterol $2011 ; 5: 378-85$.

22. Fukui T, Mitsuyama T, Takaoka M, et al. Pancreatic cancer associated with autoimmune pancreatitis in remission. Intern Med 2008;47:151-155.

23. Motosugi U, Ichikawa T, Yamaguchi H, Uchida K, Matsushita M, Okazaki K. Small invasive ductal adenocarcinoma of the pancreas associated with lymphoplasmacytic sclerosing pancreatitis. Pathol Int 2009;59:744-7.

24. Witkiewicz AK, Kennedy EP, Kennyon L, Yeo CJ, Hruban RH. Synchronous autoimmune 
pancreatitis and infiltrating pancreatic ductal adenocarcinoma: case report and review of the

literature. Hum Pathol 2008;39:1548-51.

25. Lowenfels AB, Maisonneuve P, Cavallini G, Ammann RW, Lankisch PG, Andersen JR, et al.

Pancreatitis and the risk of pancreatic cancer. International Pancreatitis Study Group. N Engl J

Med 1993;328:1433-7.

26. Chari ST, Mohan V, Pitchumoni CS, Viswanathan M, Madanagopalan N, Lowenfels AB. Risk

of pancrea tic carcinoma in tropical calcifying pancreatitis: an epidemiologic study. Pancreas

1994;9:62-6.

27. Uchida K, Yazumi S, Nishio A, Kusuda T, Koyabu M, Fukata M, et al. Long-term outcome of autoimmune pancreatitis. J Gastroenterol 2009;44:726-32.

28. Sumimoto K, Uchida K, Mitsuyama T, Fukui Y, Kusuda T, Miyoshi H, et al. A proposal of a diagnostic algorithm with validation of International Consensus Diagnostic Criteria for autoimmune pancreatitis in a Japanese cohort. Pancreatology 2013;13:230-7.

29. Shimosegawa T, Kataoka K, Kamisawa T, Miyakawa H, Ohara H, Ito T, et al. The revised Japanese clinical diagnostic criteria for chronic pancreatitis. J Gastroenterol 2010;45:584-91.

30. Hamano H, Kawa S, Horiuchi A, Unno H, Furuya N, Akamatsu T, et al. High serum IgG4 
concentrations in patients with sclerosing pancreatitis. N Engl J Med 2001;344:732-8.

31. Matsushita M, Yamashina M, Ikeura T, Shimatani M, Uchida K, Takaoka M, et al. Effective steroid pulse therapy for the biliary stenosis caused by autoimmune pancreatitis. Am J Gastroenterol 2007;102:220-1.

32. Tomiyama T, Uchida K, Matsushita M, Ikeura T, Fukui T, Takaoka M, et al. Comparison of steroid pulse therapy and conventional oral steroid therapy as initial treatment for autoimmune pancreatitis. J Gastroenterol 2011;46:696-704.

33. Koike K. Hepatocarcinogenesis in hepatitis viral infection: lessons from transgenic mouse studies. J Gastroenterol 2002;37 Suppl 13:55-64.

34. Asaka M, Takeda H, Sugiyama T, Kato M. What role does Helicobacter pylori play in gastric cancer? Gastroenterology 1997;113:S56-60.

35. Eaden JA, Abrams KR, Mayberry JF. The risk of colorectal cancer in ulcerative colitis: a meta-analysis. Gut 2001;48:526-35.

36. Kubota K, Iida H, Fujisawa T, Yoneda M, Inamori M, Abe Y, et al. Clinical factors predictive of spontaneous remission or relapse in cases of autoimmune pancreatitis. Gastrointest Endosc 2007;66:1142-51. 
autoimmune pancreatitis: characteristics of chronic disease and recurrence. Clin Gastroenterol

Hepatol 2009;7:S18-22.

38. Maruyama M, Arakura N, Ozaki Y, Watanabe T, Ito T, Yoneda S, et al. Type 1 autoimmune pancreatitis can transform into chronic pancreatitis: a long-term follow-up study of 73 Japanese patients. Int J Rheumatol 2013;2013:272595.

39. Almoguera C, Shibata D, Forrester K, Martin J, Arnheim N, Perucho M. Most human carcinomas of the exocrine pancreas contain mutant c-K-ras genes. Cell 1988;53:549-54.

40. Shiokawa M, Kodama Y, Yoshimura K, Kawanami C, Mimura J, Yamashita Y, et al. Risk of cancer in patients with autoimmune pancreatitis. Am J Gastroenterol 2013;108:610-17.

41. Tai P, Yu E, Joseph K, Miale T. A review of autoimmune diseases associated with cancer. Front Biosci (Elite Ed) 2010;2:122-6.

42. Suda K, Mogaki M, Oyama T, Matsumoto Y. Histopathologic and immunohistochemical studies on alcoholic pancreatitis and chronic obstructive pancreatitis: special emphasis on ductal obstruction and genesis of pancreatitis. Am J Gastroenterol 1990;85:271-6.

43. Hart PA, Kamisawa T, Brugge WR, Chung JB, Culver EL, Czakó L, et al. Long-term outcomes 
of autoimmune pancreatitis: a multicentre, international analysis. Gut 2013;62:1771-6.

44. Nishino T, Toki F, Oyama H, Shimizu K, Shiratori K. Long-term outcome of autoimmune pancreatitis after oral prednisolone therapy. Intern Med 2006;45:497-501.

45. Egawa S, Toma H, Ohigashi H, Okusaka T, Nakao A, Hatori T, et al. Japan Pancreatic Cancer Registry; 30th year anniversary: Japan Pancreas Society. Pancreas 2012;41:985-92.

46. Zhang L, Notohara K, Levy MJ, Chari ST, Smyrk TC. IgG4-positive plasma cell infiltration in the diagnosis of autoimmune pancreatitis. Mod Pathol 2007;20:23-8.

47. Bang SJ, Kim MH, Kim dH, Lee TY, Kwon S, Oh HC, et al. Is pancreatic core biopsy sufficient to diagnose autoimmune chronic pancreatitis? Pancreas 2008;36:84-9.

48. Fukui Y, Uchida K, Sumimoto K, Kusuda T, Miyoshi H, Koyabu M, et al. The similarity of Type 1 autoimmune pancreatitis to pancreatic ductal adenocarcinoma with significant IgG4-positive plasma cell infiltration. J Gastroenterol 2013;48:751-61.

49. Moon SH, Kim MH, Park DH, Hwang CY, Park SJ, Lee SS, et al. Is a 2-week steroid trial after initial negative investigation for malignancy useful in differentiating autoimmune pancreatitis from pancreatic cancer? A prospective outcome study. Gut 2008;57:1704-12. 


\section{FIGURE LEGEND}

Fig. 1

(a) CT revealed a focal enlargement in the head of pancreas (arrow). (b) Steroid therapy led to marked improvement of pancreatic enlargement. (c) While being treated with maintenance steroid therapy, a low-density mass in the pancreatic head was detected (arrowhead).

Fig. 2

Histological findings of Case 1. (a) Histological examination revealed a moderate-differentiated ductal adenocarcinoma (H\&E, $\times 100)$. (b) Marked lymphoplasmacytic infiltration with storiform fibrosis was observed around the PaC area (H\&E, $\times 100)$. (c) Immunohistochemically, abundant IgG4-positive cells were detected $(\mathrm{IgG} 4, \times 100)$. (d) PanIN lesion was absent in the non-cancerous region surrounding the PaC $(\mathrm{H} \& \mathrm{E}, \times 400)$.

Fig. 3

Histological findings of Case 2. (a) Histological examination revealed a moderate-differentiated ductal 
adenocarcinoma (H\&E, $\times 100)$. (b) Massive fibrosis with acinar atrophy and less severe infiltration of lymphocytes were observed around the PaC area (H\&E, ×100). (c) Scant IgG4-positive cells were detected (IgG4, $\times 100)$. (d) PanIN lesion was absent in the non-cancerous region surrounding the PaC $(\mathrm{H} \& \mathrm{E}, \times 400)$.

Fig. 4

Figure 4. (a) MRI showed a focal enlargement in the head of the pancreas (arrow). (b) Steroid therapy led to improvement of pancreatic enlargement. (c) While being treated with maintenance steroid therapy, a pancreatic body tumor with upstream dilatation of the pancreatic duct was detected (arrowhead). 
Table 1. Characteristics of patients with type 1 AIP

\begin{tabular}{|c|c|}
\hline & All patients $(\mathrm{N}=63)$ \\
\hline Male sex & $45(71 \%)$ \\
\hline Mean age at AIP diagnosis, $y$ & $62.6(19-80)$ \\
\hline Mean observation period, mo & $62.4(12-195)$ \\
\hline Drinkers & $5(8 \%)$ \\
\hline Smokers & $26(41 \%)$ \\
\hline Diabetes & $22(35 \%)$ \\
\hline \multicolumn{2}{|l|}{ Enlargement of pancreas } \\
\hline Diffuse & 20) \\
\hline Focal & $30(48 \%)$ \\
\hline None & $7(11 \%)$ \\
\hline \multicolumn{2}{|l|}{ Narrowing of MPD } \\
\hline Long or multiple & 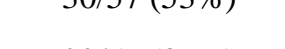 \\
\hline Focal & $22 / 57(39 \%)$ \\
\hline None & $5 / 57(8 \%)$ \\
\hline \multicolumn{2}{|l|}{ Elevated serum IgG4 } \\
\hline$>2 \mathrm{x}$ upper limit of normal value & $40 / 61(66 \%)$ \\
\hline $1-2 x$ upper limit of normal value & $17 / 61(28 \%)$ \\
\hline None & $4 / 61(6 \%)$ \\
\hline \multicolumn{2}{|l|}{ OOI } \\
\hline Sclerosing cholangitis & $9(14 \%)$ \\
\hline Enlarged salivary/lachrymal glands & $7(11 \%)$ \\
\hline Retroperitoneal fibrosis & $5(8 \%)$ \\
\hline Renal involvement & $3(5 \%)$ \\
\hline Initial therapy & \\
\hline & $51(81 \%)$ \\
\hline Steroid & $7(11 \%)$ \\
\hline Resection & $5(8 \%)$ \\
\hline Conservative follow-up & \\
\hline Maintenance steroid therapy & $39 / 51(76 \%)$ \\
\hline Relapse & $12(19 \%)$ \\
\hline \multicolumn{2}{|l|}{ Development of malignancies } \\
\hline Pancreatic cancer & $3(5 \%)$ \\
\hline Gastric cancer & $2(3 \%)$ \\
\hline Colon cancer & $1(2 \%)$ \\
\hline Leukemia & $1(2 \%)$ \\
\hline Melanoma & $1(2 \%)$ \\
\hline
\end{tabular}


Table 2. Clinical characteristics of type 1 AIP patients with or without development of PaC during follow-up

\begin{tabular}{|c|c|c|c|}
\hline & $\begin{array}{l}\text { AIP patients } \\
\text { with } \mathrm{PaC} \\
\quad(\mathrm{n}=3)\end{array}$ & $\begin{array}{l}\text { AIP patients } \\
\text { without } \mathrm{PaC} \\
\quad(\mathrm{n}=60)\end{array}$ & $\mathrm{p}$ \\
\hline Male sex & $1(33 \%)$ & $44(73 \%)$ & 0.193 \\
\hline Mean age at AIP diagnosis, $y$ & $59(30-77)$ & $62.8(19-80)$ & 0.662 \\
\hline Mean observation period, mo & $94.7(31-186)$ & $60.8(12-195)$ & 0.561 \\
\hline Drinkers & 0 & $5(8 \%)$ & 1.000 \\
\hline Smokers & $1(33 \%)$ & $25(42 \%)$ & 1.000 \\
\hline Diabetes & $1(33 \%)$ & $21(35 \%)$ & 1.000 \\
\hline Enlargement of pancreas & 0 & $26(43 \%)$ & \\
\hline Diffuse & $3(100 \%)$ & $27(45 \%)$ & 0.102 \\
\hline $\begin{array}{l}\text { Focal } \\
\text { None }\end{array}$ & 0 & $7(12 \%)$ & \\
\hline Narrowing of MPD & 0 & $30 / 54(56 \%)$ & \\
\hline Long or multiple & $3(100 \%)$ & $19 / 54(35 \%)$ & 0.053 \\
\hline $\begin{array}{l}\text { Focal } \\
\text { None }\end{array}$ & 0 & $5 / 54(9 \%)$ & \\
\hline Elevated serum IgG4 & & & \\
\hline$>2 x$ upper limit of normal value & $3(100 \%)$ & $37 / 58(64 \%)$ & \\
\hline $1-2 x$ upper limit of normal value & 0 & $17 / 58(29 \%)$ & 0.544 \\
\hline None & 0 & $4 / 58(7 \%)$ & \\
\hline OOI & 0 & $19(33 \%)$ & 0.547 \\
\hline Initial therapy & $3(100 \%)$ & $48(80 \%)$ & \\
\hline Resection & 0 & $7(12 \%)$ & 1.000 \\
\hline Maintenance steroid therapy & $3(100 \%)$ & $36 / 48(75 \%)$ & 0.439 \\
\hline Relapse & 0 & $12(20 \%)$ & 1.000 \\
\hline
\end{tabular}


Table 3. Comparison in incidence of $\mathrm{PaC}$ between patients with type $1 \mathrm{AIP}$ and $\mathrm{CP}$

\begin{tabular}{lccc}
\hline & Type 1 AIP (N=63) & CP (N=41) & $\mathrm{p}$ \\
\hline Male sex & $45(71 \%)$ & $29(73 \%)$ & 0.938 \\
Mean age at diagnosis, y & $62.6(19-80)$ & $60.2(22-89)$ & 0.185 \\
Mean observation period, mo & $62.4(12-195)$ & $49.2(13-88)$ & 0.085 \\
Drinkers & $5(8 \%)$ & $25(61 \%)$ & $<0.001$ \\
Smokers & $26(41 \%)$ & $21(51 \%)$ & 0.319 \\
Incidence of $\mathrm{PaC}$ & $3(4.8 \%)$ & $1(2.4 \%)$ & 1.000 \\
\hline
\end{tabular}



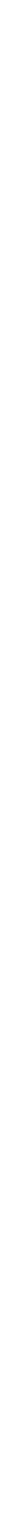

.

.

.

.

.

.

.
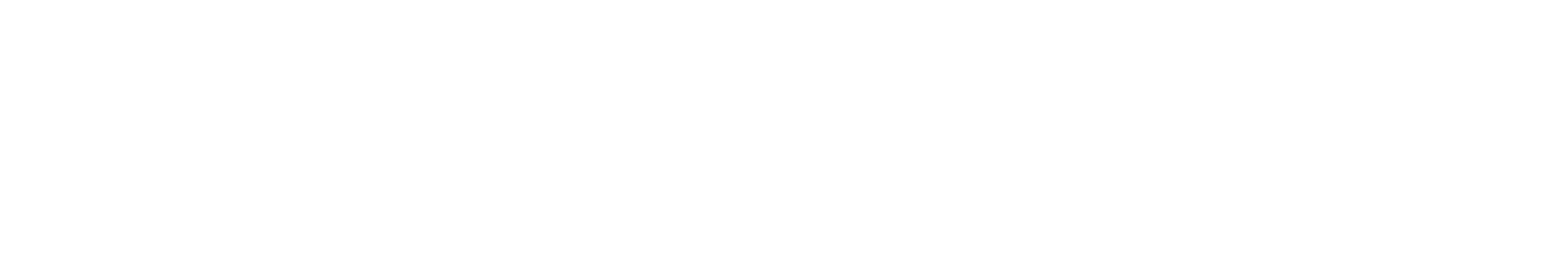

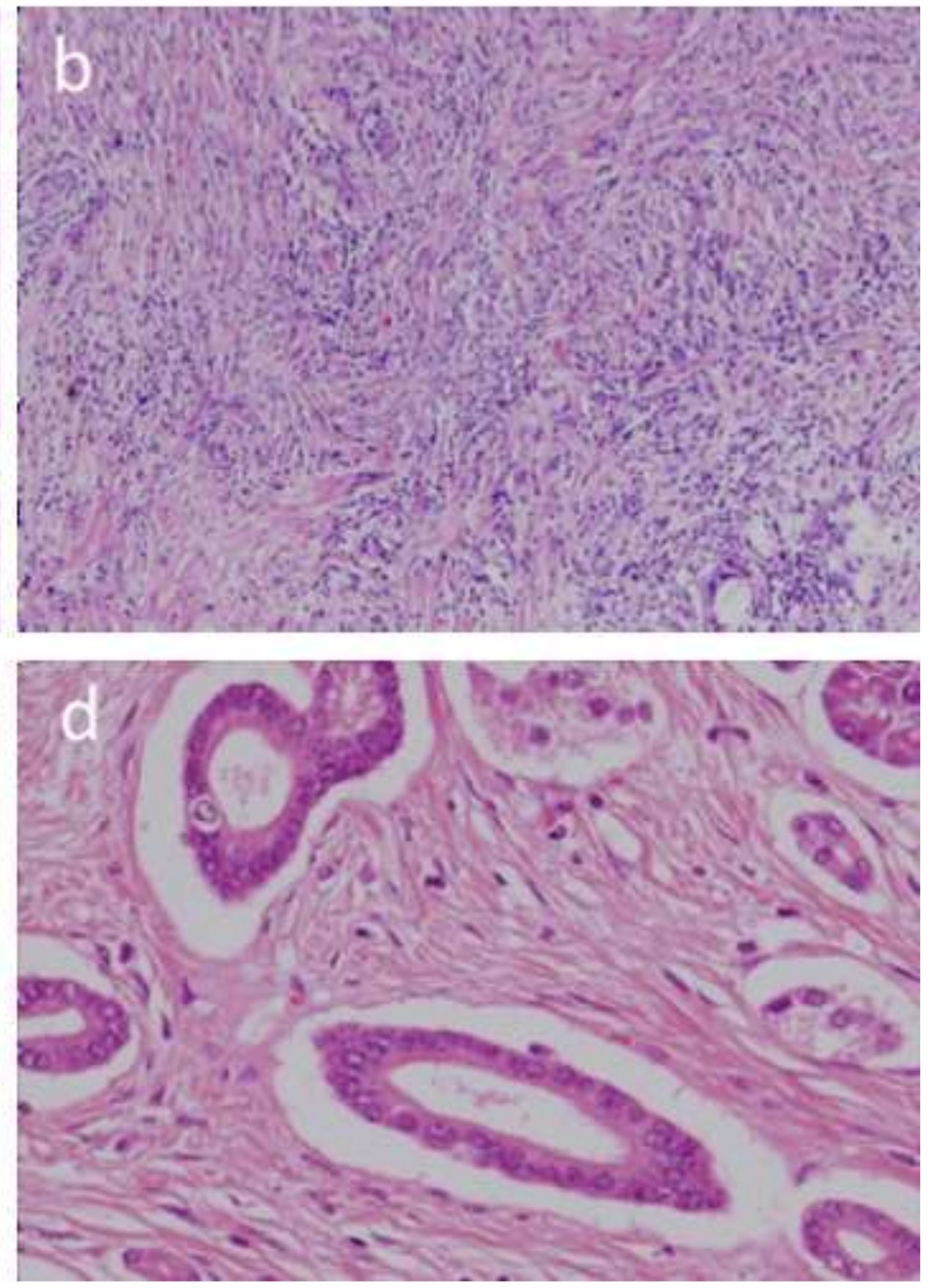
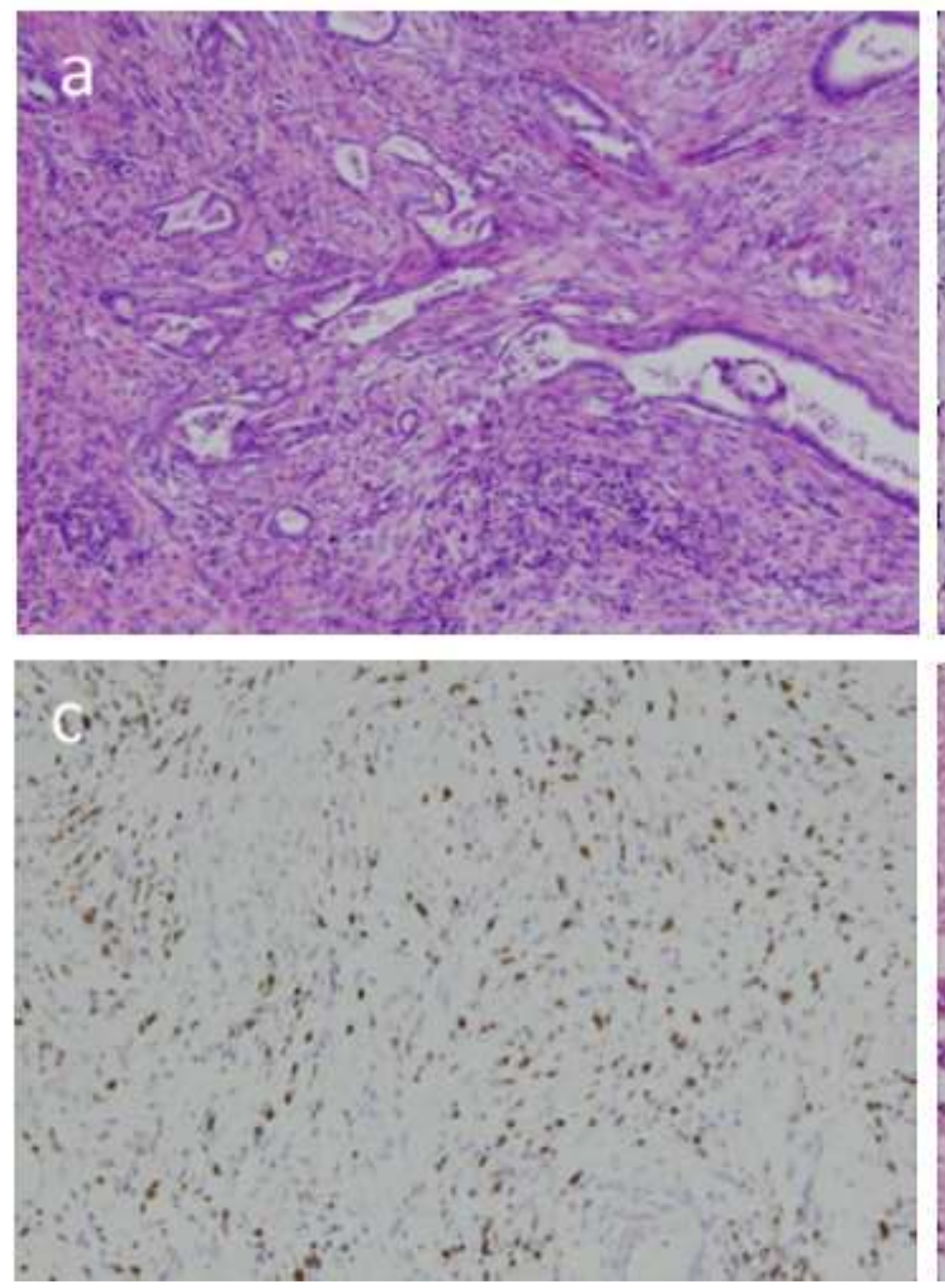

sa

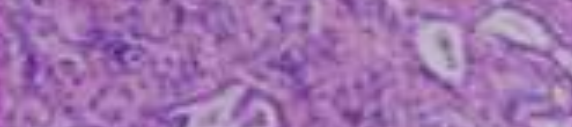

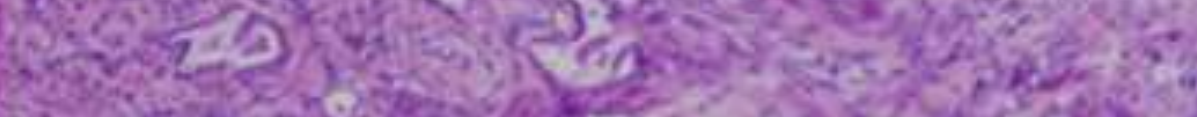

Wer

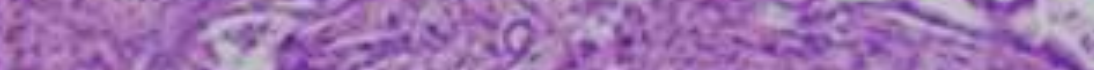

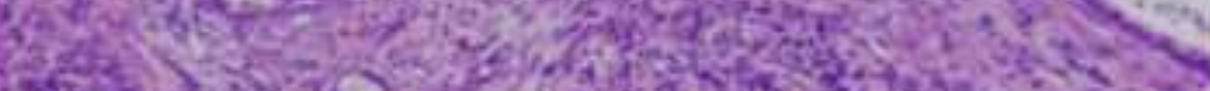

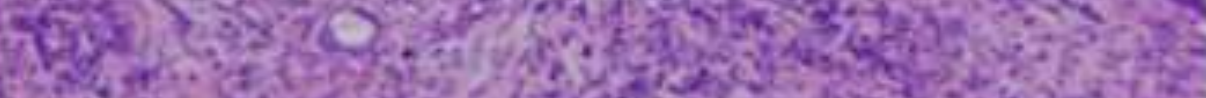

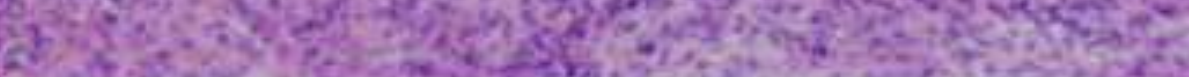



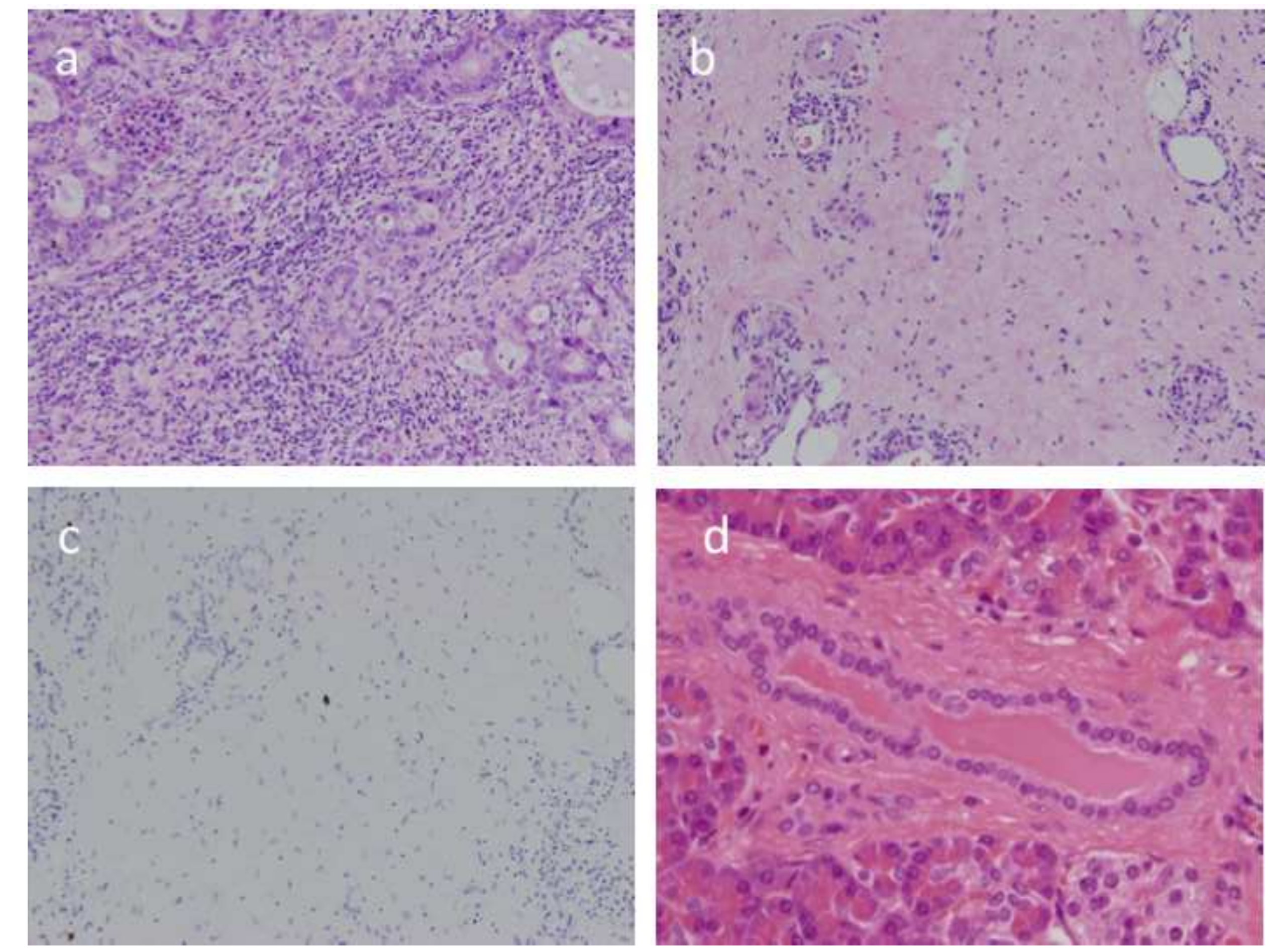


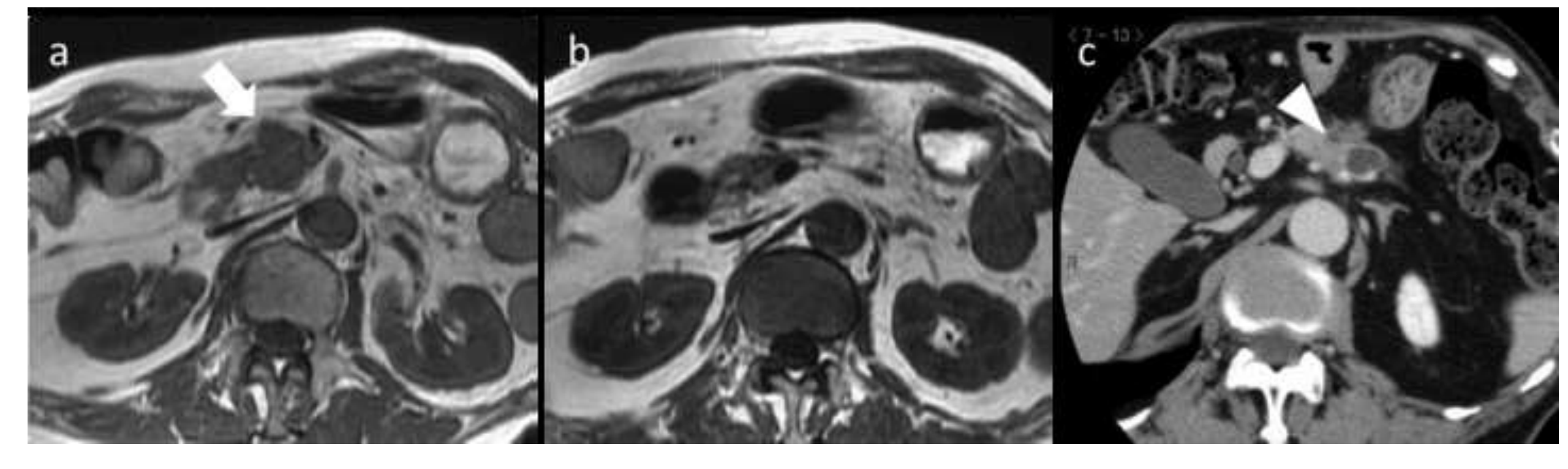

.
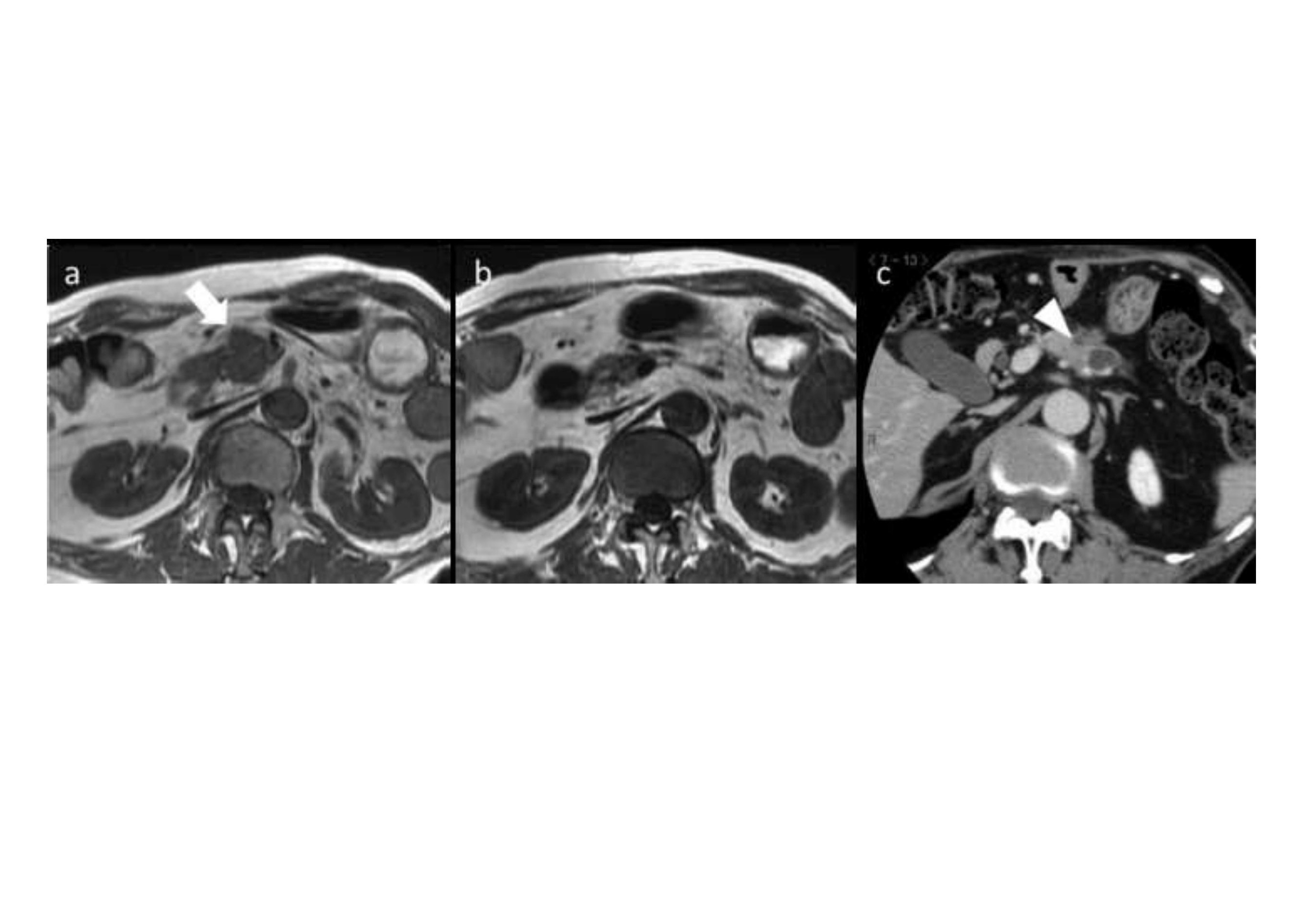